\title{
ESTUDO COMPARATIVO DE ISOLADOS DE Colletotrichum graminicola ATRAVÉS DA ANÁLISE ELETROFORÉTICA DE PADRÕES PROTÉICOS E ISOENZIMÁTICOS*
}

\author{
MARIA L.F. LIMA \& MARIA MENEZES ${ }^{2}$ \\ Fitossanidade - Departamento de Agronomia - Universidade Federal Rural de Pernambuco. \\ Rua Manoel de Medeiros, S/N - Dois Irmãos, 52171-900, Recife/PE \\ (Aceito para publicação em 13/11/2001)
}

Autor para correspondência: Maria do Livramento Ferreira Lima

LIMA, M.L.F. \& MENEZES, M. Estudo comparativo de isolados de Colletotrichum graminicola através da análise eletroforética de padrões protéicos e isoenzimáticos. Fitopatologia Brasileira 27:012-016. 2002.

\section{RESUMO}

Colletotrichum graminicola, agente causal da antracnose ou podridão do colmo do milho (Zea mays), é um importante patógeno com alta variabilidade genética. Nove isolados desta espécie foram comparados através do uso da análise eletroforética em gel de poliacrilamida, utilizando três sistemas: proteínas totais, esterase e fosfatase ácida. A análise mostrou variação no número e posição das bandas no gel, dentro de cada sistema estudado. Com relação a proteínas totais, foi observado maior polimorfismo, embora os isolados Cgr8 e Cgr9 tenham mostrado idêntico perfil com seis bandas protéicas de mesma mobilidade relativa. Na atividade esterásica, esses mesmos isolados apresentaram um comportamento monomórfico, enquanto os demais revelaram polimorfismo. $\mathrm{Na}$ análise fosfatase ácida, todos os isolados mostraram-se semelhantes quanto à presença de duas bandas, porém diferentes em relação a mobilidade das moléculas no gel. Igualdade de comportamento, quanto à mobilidade relativa das bandas de fosfatase ácida, foi observada entre Cgr1 e Cgr2, como também entre Cgr6 , Cgr7 e Cgr8. Em geral, nos três sistemas testados, os isolados de $C$. graminicola apresentaram bandas em comum, indicando a relação existente entre eles, e que a variação fenotípica observada seja provavelmente decorrente da condição nuclear, homozigótica ou heterozigótica, de cada isolado do fitopatógeno.

Palavras-chave adicionais: antracnose, Zea mays, isoenzima.

\section{ABSTRACT \\ A comparative study of Colletotrichum graminicola isolates by electroforetic analysis of proteins and isozymes}

Colletotrichum graminicola, the causal agent of anthracnose or stalk rot of corn (Zea mays), is an important pathogen with a high genetic variability. Nine isolates of this phytopathogen were compared by using isozyme analysis with polyacrylamide gel electrophoresis in three systems: proteins, esterase and acidic phosphatase. Analysis by each system showed variation in the number, intensity, and relative mobility of the bands in the gel of the pathogen population. For protein the analysis revealed polymorphism eventhough Cgr8 and Cgr9 isolates have shown the same pattern with six bands of relative position in the

\section{INTRODUÇÃO}

Colletotrichum graminicola (Ces.) G. W. Wilson (Teleomorfo: Glomerella graminicola Politis) é um importante patógeno, causando antracnose ou podridão do colmo em várias gramíneas. Economicamente os hospedeiros mais importantes são o milho (Zea mays L.) e o sorgo (Sorghum bicolor) (Vaillancourt \& Hanau, 1992; Casela et al., 1998). A podridão do colmo é o maior fator limitante da produção de milho no mundo (Keller \& Bergstrom, 1988). A doença é

\footnotetext{
* Parte da dissertação do primeiro autor. Universidade Federal Rural de Pernambuco (2000). Financiado pela CAPES.
}

gel. For esterase activity, the same isolates showed a monomorphic behaviour while the other isolates revealed polymorphic phenotypes. In relation to acidic phosphatase, all isolates presented two bands, but differences in mobility of the molecules were observed, except between Cgr1 and Cgr2 and among Cgr6, Cgr7 and Cgr8. In general, the isolates produced bands in common, indicating that they are closely related to each other, and that the phenotypic variation observed, is probably the result of a nuclear condition, homozygous or heterozygous, for each enzyme system tested. problemática e todos os tecidos da planta são afetados e as várias fases dos sintomas ocorrem durante a estação de cultivo (Bailey \& Jeger, 1992).

$\mathrm{O}$ agente causal da antracnose do milho possui uma alta variabilidade, dificultando a obtenção de cultivares resistentes (Guthrie et al.,1992). O sucesso de um programa de melhoramento, visando resistência a doenças, depende do conhecimento da extensão da diversidade genética dentro da população do agente causal. Atualmente, vários métodos são utilizados para identificar de forma rápida e precisa a variação genética dentro de uma mesma espécie, como o uso de marcadores moleculares (Crowhurst et al., 1991; Guzmán et al., 1995) e, dentre estes métodos, são citados as isoenzimas. 
Estudo comparativo de isolados de Colletotrichum graminicola através da análise...

Padrões eletroforéticos de isoenzimas representam uma expressão direta do genoma de um organismo, podendo ser de valor considerável na determinação da variação entre grande número de locos de enzimas (Micales et al., 1986; Boshoff et al., 1996). Este conhecimento serve também de base para estudos taxonômicos, como complemento aos marcadores morfológicos, tradicionalmente utilizados na identificação de fitopatógenos, permitindo estimar quantitativamente a variabilidade de diferentes organismos, dentre eles, os fitopatógenos (Reynolds et al., 1983; McDonald \& McDermott, 1993). As isoenzimas constituem ainda um método adicional na diferenciação de formas especiais e/ou raças fisiológicas dentro de espécies fúngicas, muito bem documentado por vários pesquisadores (Burdon \& Marshall, 1981; Petrunak \& Christ, 1992; Figueiredo et al., 1993; Rego et al., 1994). Outros têm estudado a atuação de enzimas extracelulares e intracelulares na patogenicidade de fungos (Hancock \& Millar, 1965; Bosland \& Williams, 1987; Bonde et al., 1993). Também, Lenné \& Burdon (1990) investigaram sobre a virulência e variação enzimática de Colletotrichum gloeosporioides (Penzig) Penzig \& Saccardo, verificando que $80 \%$ dos isolados, apresentando o mesmo fenótipo para virulência, mostraram fenótipos isoenzimáticos com diferentes locos gênicos.

O propósito do presente trabalho foi comparar o perfil de proteínas e isoenzimas de nove isolados de C. graminicola, através da análise eletroforética em gel de poliacrilamida, e avaliar esta análise como um método para diferenciação intraespecífica.

\section{MATERIAL E MÉTODOS}

Nove isolados de C. graminicola oriundos de milho cultivado em diferentes regiões do Brasil (Cgr1, UberlândiaMG; Cgr2, Dourados-MS; Cgr3, Santa Helena-GO; Cgr4, Sete Lagoas-MG; Cgr5, Palotina-PR; Cgr6, Londrina-PR; Cgr7, Cascavel-PR; Cgr8, Cristalina-GO; Cgr9, Sete Lagoas-MG), foram cultivados em $60 \mathrm{ml}$ de meio líquido batata-dextrose (BD), durante cinco dias, a $25^{\circ} \mathrm{C}$, em regime de luz contínua. Após o período de incubação a massa micelial foi coletada por filtração, lavada com água destilada e esterilizada, sendo o excesso de umidade retirado com auxílio de papel de filtro. Em seguida, $500 \mathrm{mg}$ do micélio foram macerados em banho de gelo, adicionando-se à massa micelial, $1 \mathrm{ml}$ de tampão Tris-glicina $(0,125 \mathrm{M}, \mathrm{pH} 8,2)$; $300 \mathrm{mg}$ de polivinilpirrolidona; $300 \mathrm{mg}$ de sacarose, para obtenção dos extratos protéicos.

Em geral, para as análises eletroforéticas foi seguida a metodologia descrita por Alfenas et al. (1991, 1998). Placas de gel de poliacrilamida a $5 \%$ foram preparadas através da mistura de acrilamida e bisacrilamida em solução tampão Tris-glicina $(0,125 \mathrm{M}, \mathrm{pH} 8,2)$ com adição de $0,1 \mathrm{ml}$ de tetrametildiamina (TEMED) e 2,8 $\mathrm{ml}$ de persulfato de amônio a $1 \%$. Após a polimerização do gel, $20 \mu \mathrm{l}$ do extrato micelial dos isolados foram colocados em cada cavidade do gel. A corrida eletroforética foi realizada a $4{ }^{\circ} \mathrm{C}$, mantendo-se a corrente constante a $10 \mathrm{~mA}$, em cuba horizontal, contendo tampão Trisglicina $0,125 \mathrm{M}, \mathrm{pH} 8,2$. O azul de bromofenol foi o corante marcador utilizado e a migração ocorreu no período de $4 \mathrm{~h}$.

Para detecção de proteínas totais, após a corrida eletroforética, o gel foi imerso durante $12 \mathrm{~h}$ numa solução corante preparada com Comassie blue R-250, 100 mg; metanol, $45 \mathrm{ml}$; ácido acético, $10 \mathrm{ml}$; água destilada, $45 \mathrm{ml}$. Após a formação das bandas de proteínas o excesso de corante foi removido por lavagens sucessivas do gel, em solução clareadora preparada com metanol, $45 \mathrm{ml}$; ácido acético glacial, $10 \mathrm{ml}$; água destilada, $45 \mathrm{ml}$.

Para detecção de bandas de esterase, o gel foi imerso por uma hora em uma solução corante, composta de tampão fosfato, $100 \mathrm{ml}$ a $0,1 \mathrm{M} \mathrm{pH} \mathrm{6,5;} \mathrm{alfa-naftil} \mathrm{acetato,} 50 \mathrm{mg}$ a $1 \%$; fast blue RR, $50 \mathrm{mg} ; 2,5 \mathrm{ml}$ de acetona, $2,5 \mathrm{ml}$ de água destilada, em condições de escuro.

Para detecção de fosfatase ácida, o gel foi imerso na solução de Fast Garnet e deixado em ambiente escuro a $37^{\circ} \mathrm{C}$ até o aparecimento das bandas. A solução corante foi preparada com $\alpha$-naftil fosfato ácido de sódio, $100 \mathrm{mg}$; fast garnet $\mathrm{GBC}$, $100 \mathrm{mg} ; \mathrm{Mg} \mathrm{Cl}_{2}$ (1\%), $1 \mathrm{ml}$; tampão acetato de sódio $(0,1 \mathrm{M}$, $\mathrm{pH} \mathrm{5,0),} 100 \mathrm{ml}$.

As avaliações para diferenciação protéica e isoenzimática foram efetuadas com base no número, intensidade $\mathrm{e}$ mobilidade relativa das bandas formadas nos géis de poliacrilamida. $\mathrm{O}$ cálculo da mobilidade relativa das bandas foi efetuado pela fórmula descrita por Alfenas et al. (1991): $\mathrm{Rf}=$ $(d / D) \times 100$, onde: $d=$ distância percorrida pela molécula ; D = distância percorrida pelo corante (controle). Os dados obtidos foram analisados pelo uso do método UPGMA (Unweighted Pair Group Method with Arithmetic Mean) e os resultados expressos em dendrogramas.

\section{RESULTADOS E DISCUSSÃO}

O procedimento seguido na execução deste trabalho permitiu detectar, em todos os isolados de C. graminicola, fenótipos eletroforéticos de proteínas, isoesterase e fosfatase ácida.

Com relação a produção de bandas protéicas, os isolados analisados apresentaram comportamento polimórfico. Em geral, o número de bandas produzidas variou de seis a oito, com predominância de seis, em $67 \%$ dos isolados, com intensidade forte, média e fraca, observadas em várias regiões do gel. Dos nove isolados, somente Cgr8 e Cgr9 mostraram estreita semelhança entre si, evidenciada pelo número, intensidade e mesma mobilidade das bandas protéicas, no gel de poliacrilamida. As enzimas fúngicas são semelhantes às de outros organismos, consistindo de proteínas e cofatores associados que atuam nos processos metabólicos. Griffin (1994) ressalta o papel importante das enzimas extracelulares na patogênese, permitindo a penetração e colonização do tecido hospedeiro, sendo o grupo das celulases o mais extensivamente estudado. Embora não tenha sido este o objetivo da presente pesquisa, o fato mostra a importância da utilização de vários sistemas para detecção das enzimas produzidas pelos 
fungos fitopatogênicos, permitindo a diferenciação a níveis subespecíficos, difícil de ser observada através de marcadores morfológicos. Estes mesmos isolados apresentaram fenótipos iguais com relação a esterase, com apenas uma banda monomórfica, de intensidade fraca e mesma localização no gel. Em adição, estes dois isolados de C. graminicola, em estudos prévios, comportaram-se como os mais patogênicos em relação aos demais, quando inoculados em três cultivares de milho. No sistema fosfatase ácida, todos os isolados apresentaram duas bandas da enzima com variação na intensidade e posição no gel.

Com exceção do sistema fosfatase ácida, a maioria dos isolados mostrou pequeno polimorfismo esterásico e protéico, sendo mais acentuado neste último. Dentro de cada sistema testado, foram constatadas bandas em comum produzidas pelos isolados e localizadas em diferentes regiões dos géis.

Figueiredo et al. (1989) investigaram a variabilidade isoenzimática de 15 isolados de $C$. lindemuthianum, através da eletroforese em gel de amido, e detectaram atividade para 20 enzimas, das 25 testadas, sendo a maioria delas polimórficas. Também Véras et al. (1997) avaliaram nove isolados de $C$. guaranicola, usando os mesmos sistemas utilizados no presente trabalho, tendo também observado variações fenotípicas entre os isolados, embora pertencentes a mesma espécie.

De acordo com os valores da mobilidade relativa (Rf), das bandas protéicas e isoenzimáticas, nos três sistemas analisados (Tabelas 1, 2, 3). Além do tamanho da molécula, outros fatores podem estar envolvidos na diferença de mobilidade das bandas observadas nos isolados de C. graminicola.
Considerando Micales et al. (1986) e Bonde et al. (1993), as enzimas que são codificadas por locos ou alelos diferentes, freqüentemente mostram diferenças na mobilidade relativa das bandas.

A análise de agrupamento usada para estimar os dados em relação a atividade protéica e isoenzimática dos isolados de C. graminicola, tomando-se como base a mobilidade relativa das bandas no gel de poliacrilamida, revelou uma variabilidade intraespecífica, de acordo com o sistema empregado (Figuras 1, 2 e 3). Em geral, os agrupamentos são condicionados pela alta freqüência de fatores complementares na população do patógeno.

No sistema protéico (Figura 1), a análise dos isolados permitiu detectar seis grupos: 1(Cgr1), 2 (Cgr2 e Cgr3), 3 (Cgr4), 4 (Cgr5 e Cgr6), 5 (Cgr7) e 6(Cgr8 e Cgr9), os quais diferiram na expressão da similaridade. Ao nível de $0 \%$ de discordância, os isolados Cgr8 e Cgr9 mostraram-se idênticos entre si, enquanto Cgr1 foi o mais discordante de todos (95\%).

Nos sistema esterásico (Figura 2), a análise de agrupamento dos isolados revelou sete grupos: 1 (Cgr1 e Cgr2), 2 (Cgr3), 3 (Cgr4), 4 (Cgr8 e Cgr9), 5 (Cgr6), 6 (Cgr7) e 7 (Cgr5). É interessante notar que os isolados do grupo 4 (Cgr8 e Cgr9) neste sistema revelaram também comportamento idêntico ao observado no agrupamento dos dados protéicos, ou seja, $0 \%$ de discordância. Os demais grupos apresentaram níveis de discordância acima de 40\%.

Com relação ao sistema fosfatase ácida, a análise de agrupamento dos dados permitiu definir cinco grupos: 1(Cgr1 e Cgr2), 2 (Cgr3), 3 (Cgr6, Cgr7 e Cgr8), 4 (Cgr4) e 5 (Cgr5 e Cgr9), dos quais os isolados incluídos nos grupos 1, 3 e 5

TABELA 1 - Intensidade e mobilidade relativa das bandas de proteínas totais apresentadas pelos isolados de Colletotrichum graminicola, em gel de poliacrilamida

\begin{tabular}{|c|c|c|c|c|c|c|c|c|c|c|c|c|c|c|c|c|c|c|c|c|c|c|c|c|c|}
\hline \multirow{2}{*}{ Isolado } & \multirow{2}{*}{$\mathbf{N}^{\circ}$ Bandas } & \multicolumn{3}{|c|}{ Intens. das Bandas } & \multirow[b]{2}{*}{1} & \multirow[b]{2}{*}{2} & \multirow[b]{2}{*}{3} & \multicolumn{18}{|c|}{ "Mobilidade Relativa (Rf) } \\
\hline & & Forte & Média & Fraca & & & & 4 & 5 & 6 & 7 & 8 & 9 & 10 & 11 & 12 & 13 & 14 & 15 & 16 & 17 & 18 & 19 & 20 & 21 \\
\hline Cgr1 & 6 & 1 & 3 & 2 & & & & 15,0 & & 25,0 & & & & & 38,3 & & & 45,0 & & 55,0 & & & & 73,3 & \\
\hline Cgr2 & 7 & 1 & 3 & 3 & & & & 15,0 & 23,3 & & & 30,0 & & & & 41,7 & & & & 55,0 & & 66,7 & & & 75,0 \\
\hline Cgr3 & 8 & 5 & 1 & 2 & 6,7 & & & 15,0 & 23,3 & & & & 35,0 & & & 41,7 & & & & 55,0 & & 66,7 & & & 75,0 \\
\hline Cgr4 & 7 & 7 & & & & 11,7 & & & 23,3 & & & & & 36,7 & & & 43,3 & & & 55,0 & & 66,7 & & & 75,0 \\
\hline Cgr5 & 6 & 1 & 2 & 3 & & 11,7 & & & 23,3 & & 28,3 & & & & & 41.7 & & & & 55,0 & & & & 73,3 & \\
\hline Cgr6 & 6 & 5 & 1 & - & & 11,7 & & & 23,3 & & 28,3 & & & 36,7 & & & & & & 55,0 & & & & 73,3 & \\
\hline Cgr7 & 6 & 3 & 3 & - & & & & & 23,3 & & 28,3 & & & 36,7 & & & & & & 55,0 & & & 68,3 & 73,3 & \\
\hline Cgr8 & 6 & & & 6 & & & 13,3 & & 23,3 & & & & 35,0 & & & & & & 51,7 & & 56,7 & & & 73,3 & \\
\hline Cgr9 & 6 & - & & 6 & & & 13,3 & & 23,3 & & & & 35,0 & & & & & & 51,7 & & 56,7 & & & 73,3 & \\
\hline
\end{tabular}

TABELA 2 - Intensidade e mobilidade relativa das bandas de esterase apresentadas pelos isolados de Colletotrichum graminicola, em gel de poliacrilamida

\begin{tabular}{|c|c|c|c|c|c|c|c|c|c|c|c|c|c|c|c|c|c|c|c|c|}
\hline \multirow{2}{*}{ Isolado } & \multirow{2}{*}{$\mathbf{N}^{\circ}$ Bandas } & \multicolumn{3}{|c|}{ Intens.das Bandas } & \multicolumn{16}{|c|}{ "Mobilidade Relativa (Rf) } \\
\hline & & Forte & Média & Fraca & 1 & 2 & 3 & 4 & 5 & 6 & 7 & 8 & 9 & 10 & 11 & 12 & 13 & 14 & 15 & 16 \\
\hline Cgr1 & 5 & 2 & - & 3 & & 54,3 & 58,6 & & & & & 68,6 & & & & 82,9 & & & & 111,1 \\
\hline Cgr2 & 3 & - & 2 & 1 & & & & 61,4 & & & & & & & & 82,9 & & & & 111,1 \\
\hline Cgr3 & 4 & 1 & 2 & 1 & & & 58,6 & & & & & 68,6 & & & 80,0 & & & & 100,0 & \\
\hline Cgr4 & 2 & 2 & - & - & & & & & & & 65,7 & & & & & & & 95,7 & & \\
\hline Cgr5 & 5 & 1 & 1 & 3 & 48,6 & & & & & 64,3 & & & & 77,1 & & & 85,7 & 95,7 & & \\
\hline Cgr6 & 4 & 3 & 1 & - & 48,6 & & & & 62,9 & & & & 75,7 & & & & & 95,7 & & \\
\hline Cgr7 & 4 & 1 & 2 & 1 & & 54,3 & & & & 64,3 & & & 75,7 & & & & & 95,7 & & \\
\hline Cgr8 & 1 & - & - & 1 & & & & & & & & & 75,7 & & & & & & & \\
\hline Cgr9 & 1 & - & - & 1 & & & & & & & & & 75,7 & & & & & & & \\
\hline
\end{tabular}


Estudo comparativo de isolados de Colletotrichum graminicola através da análise...

TABELA 3 - Intensidade e mobilidade relativa das bandas de fosfatase ácida apresentadas pelos isolados de Colletotrichum graminicola, em gel de poliacrilamida

\begin{tabular}{|c|c|c|c|c|c|c|c|c|c|}
\hline \multirow{2}{*}{ Isolado } & \multirow{2}{*}{$\mathbf{N}^{\circ}$ Bandas } & \multicolumn{3}{|c|}{ Intens. das Bandas } & \multicolumn{5}{|c|}{ Mobilidade Relativa (Rf) } \\
\hline & & Forte & Média & Fraca & 1 & 2 & 3 & 4 & 5 \\
\hline Cgr1 & 2 & 1 & 1 & - & & & 27,3 & & 45,5 \\
\hline Cgr2 & 2 & 1 & 1 & - & & & 27,3 & & 45,5 \\
\hline Cgr3 & 2 & 1 & - & 1 & & 25,5 & & & 45,5 \\
\hline $\mathrm{Cgr} 4$ & 2 & 1 & - & 1 & & 25,5 & & 44,0 & \\
\hline Cgr5 & 2 & 1 & - & 1 & 23,6 & & & 44,0 & \\
\hline Cgr6 & 2 & 1 & - & 1 & 23,6 & & & & 45,5 \\
\hline Cgr7 & 2 & 1 & 1 & - & 23,6 & & & & 45,5 \\
\hline Cgr8 & 2 & 1 & 1 & - & 23,6 & & & & 45,5 \\
\hline Cgr9 & 2 & 1 & 1 & - & 23,6 & & & 44,0 & \\
\hline
\end{tabular}

apresentaram padrões idênticos de produção e mobilidade da enzima referida, ao nível de $0 \%$ de discordância.

Sabe-se que a mobilidade das bandas protéicas e isoenzimáticas no gel está condicionada ao tamanho da molécula, constituindo os Rfs obtidos pela distância percorrida, através da eletroforese, um critério importante para identificação de diferenças e similaridade de isolados dentro de uma mesma espécie. Entretanto, vale ressaltar, que este critério não significa igualdade genética entre isolados, pois existem outros fatores a serem considerados, como exemplo, a densidade de concentração da enzima, refletida pela coloração forte e largura da banda formada, além de outros, como exemplo, a expressão da patogenicidade. Micales et al. (1986) consideram, também, que os organismos que exibem alto nível de variabilidade genética apresentam variação intraespecífica. A expressão desta variabilidade está na dependência dos sistemas enzimáticos utilizados. De acordo com Micales et al. (1998), as isoenzimas são formas moleculares múltiplas de enzimas e que somente aquelas constituidas de aminoácidos diferentes em carga elétrica ou forma podem ser separadas através da eletroforese, resultando na formação de bandas que representam apenas parte da

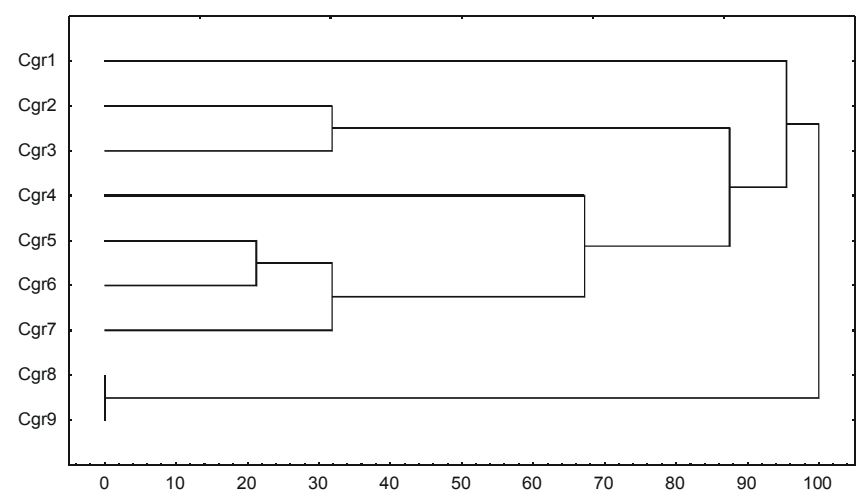

FIG. 1 - Dendrograma revelando a análise de agrupamento entre nove isolados de Colletotrichum graminicola, com base na mobilidade relativa das bandas de proteínas, no gel de poliacrilamida, pelo método UPGMA.

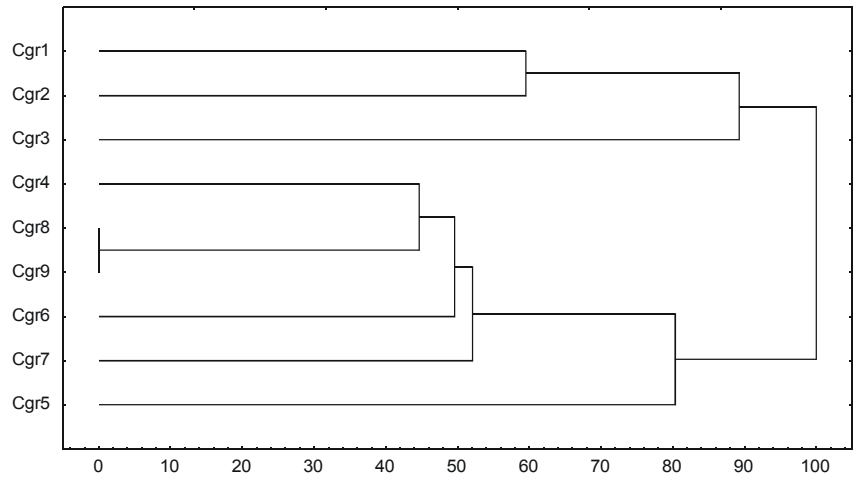

FIG. 2 - Dendrograma revelando a análise de agrupamento entre nove isolados de Colletotrichum graminicola, baseado na mobilidade relativa de bandas de esterase, pelo método UPGMA.

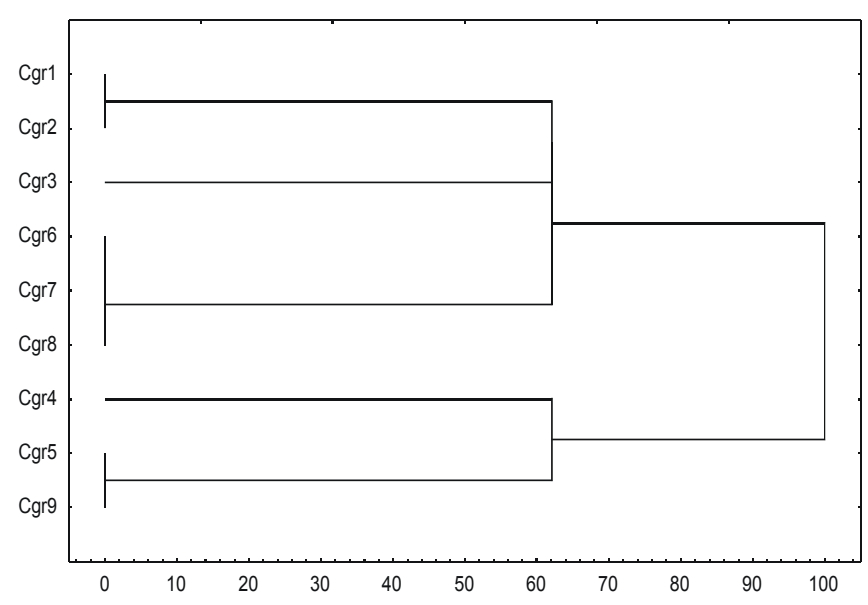

FIG. 3 - Dendrograma revelando a análise de agrupamento entre nove isolados de Colletotrichum graminicola, baseado na mobilidade relativa das bandas de fosfatase ácida, pelo método UPGMA.

variabilidade genética de um organismo. O número de bandas pode refletir o número de alelos presentes no sistema e que depende da condição nuclear (mono ou heterocariótico), do nível de ploidia (haplóide ou diplóide), da constituição genética do organismo (homozigoto ou heterozigoto), da estrutura quaternária da enzima, além de outros fatores. Embora cada isolado represente uma população de biótipos, acredita-se no entanto, que a padronização de condições de cultivo monospórico, reduza a variação observada em $C$. graminicola, quanto aos padrões protéicos e isoenzimáticos apresentados.

\section{AGRADECIMENTOS}

Os autores agradecem aos pesquisadores Carlos Roberto Casela (EMBRAPA/CNPMS) e Regina Melo Sartori Coêlho Morello (Universidade de Tocantins /UNITINS) pelo envio de isolados de C. graminicola. 


\section{REFERÊNCIAS BIBLIOGRÁFICAS}

ALFENAS, A.C., PETERS, I., BRUNE, W. \& PASSADOR, G.C. Eletroforese de Proteínas e Isoenzimas de Fungos e Essências Florestais. Viçosa. Universidade Federal de Viçosa. 1991.

ALFENAS, A.C. Eletroforese de isoenzimas e proteínas afins Fundamentos e aplicações em plantas e microrganismos. Viçosa: Universidade Federal de Viçosa. 1998.

BAILEY, J.A. \& JEGER, M.J. Colletotrichum: Biology, Pathology and Control. C.A.B International, Wallingford, UK. 1992.

BONDE, M.R., MICALES, J.A. \& PETERSON, G.L. The use of isozyme analyses for identification of plant-pathogenic fungi. Plant Disease 77:961-968. 1993.

BOSHOFF, W.H.P., SWART, W.J., PRETORIUS, Z. A., LIEBENBERG, M.M. \& CRONS, P.W. Isozyme variability among isolates of Phaeoisariopsis griseola in southern Africa. Plant Pathology 45:344-349. 1996.

BOSLAND, P.W. \& WILLIAMS, P.H. An evaluation of Fusarium oxysporum from crucifers based on pathogenicity, isozyme polymorphism, vegetative compatibility, and geografic origin. Canadian Journal of Botany 65:2065-2073. 1987

BURDON, J.J. \& MARSHALL, D.R. Isozyme variation between species and formae speciales of the genus Puccinia. Canadian Journal of Botany 59:2628-2634. 1981.

CASELA, C.R., FERREIRA, A.S. \& SANTOS, F.G. Associação de virulência de Colletotrichum graminicola à resistência genética em sorgo. Fitopatologia Brasileira 23:143-146. 1998.

CROWHURST, R.N., HAWTHOME, B.T., RIKKERINR, E.H.A. \& TEMPLETON, M.D. Differentiation of Fusarium solani $\mathrm{f}$. sp. cucurbitae races 1 and 2 by random amplification of polymorphic DNA. Current Genetics 20:391-396. 1991.

FIGUEIREDO, G., ALFENAS, A.C. \& BROMMONSCHENKEL, S.H. Variabilidade isoenzimática de Colletotrichum lindemuthianum. Fitopatologia Brasileira 14:118. 1989 (Resumo).

FIGUEIREDO, G., ALFENAS, A.C., BROMMONSCHENKEL, S.H. \& FARIA, J.C. Variabilidade isoenzimática de isolados de Colletotrichum lindemuthianum com diferentes níveis de virulência. Arquivo de Biologia e Tecnologia 36:793-808. 1993.

GRIFFIN, D.H. Fungal Physiology. $2{ }^{\text {nd }}$ Edition. John Wiley \& Sons Inc, Publication. New York. 1994.

GUTHRIE, P.A.I., MAGILL, C.W., FREDERICKSEN, R.A. \& ODVODY, G.N. Random Amplified Polymorphic DNA markers: A system for identifying and differentiating isolates of Colletotrichum graminicola. Phytopathology 82:832-835. 1992.

GUZMÁN, P., GILBERTSON, R.L., NODARI, R., JOHNSON, W.C., TEMPLE, S.R., MANDALA, D., MKANDAWIRE, A.B.C. \& GEPTS, P. Characterization of variability in the fungus Phaeoisariopsis griseola suggests coevolution with the common bean (Phaseolus vulgaris). Phytopathology 85:600607. 1995.

HANCOCK, J.G. \& MILLAR, R.L. Association of cellulolytic, proteolytic, and xylolytic enzymes with southern antracnose, spring black stem, and stemphylium leaf spot of alfafa. Phytopathology 55:356-360. 1965.

KELlER, N.P. \& BERGSTROM, G.C. Developmental predisposition of maize to anthracnose stalk rot. Plant Disease 72:977-980. 1988.

LENNÉ, J.M. \& BURDON, J.J. Preliminary study of virulence and isozyme variation in natural populations of Colletotrichum gloeosporioides from Stylosanthes guianensis. Phytopathology 80:728-731. 1990.

McDONALD, B.A. \& McDERMOTT, J.M. Population genetics of plant pathoghenic fungi. Bioscience 43:311-319. 1993.

MICALES, J.A., BONDE, M.R. \& PETERSAON, G.L. The use of isozyme analysis in fungal taxonomy and genetics. Mycotaxon 27:405-449. 1986.

MICALES, J.A., ALFENAS, A.C. \& BONDE, M.R. Isoenzimas na Taxonomia e na Genética de Fungos. In: Alfenas, A.C. (Ed.) Eletroforese de Isoenzimas e Proteínas afins. Viçosa: Universidade Federal de Viçosa. 1998. pp.477-512.

PETRUNAK, D.M. \& CHRIST, B.J. Isozyme variability in Alternaria solani and Alternaria alternata. Phytopathology 82:1343-1347. 1992.

REGO, A.M., MAFFIA, A.L. \& ALFENAS, A.C. Virulência e análise de isoenzimas de C. orbiculare. Fitopatologia Brasileira 19:552-559. 1994.

REYNOLDS, M., WEINHOLD, A.R. \& MORRIS, T.J. Comparison of anastomosis groups of Rhizoctonia solani by polyacrilamide gel electrophoresis of soluble proteins. Phytophatology 73:903906. 1983.

VAILLANCOURT, L.J. \& HANAU, R.M. Genetic and morphological comparisons of Glomerella (Colletotrichum) isolates from maize and from sorghum. Experimental Mycology 16:219-229. 1992.

VÉRAS, S.M., GASPAROTT, L. \& MENEZES, M. Avaliação isoenzimática de C. guaranicola. Arquivos de Biologia e Tecnologia 40:548-553. 1997. 